\title{
Radiation Protection Quantities for Near Earth Environments
}

\author{
Martha S. Clowdsley and John W. Wilson \\ NASA Langley Research Center, Hampton, Virginia, 23681 \\ Myung-Hee Kim \\ Wyle Laboratories, Houston, Texas, 77058 \\ Brooke M. Anderson \\ NASA Langley Research Center, Hampton, Virginia, 23681 \\ and \\ John E. Nealy \\ Old Dominion University, Norfolk, Virginia, 23508
}

\begin{abstract}
As humans travel beyond the protection of the Earth's magnetic field and mission durations grow, risk due to radiation exposure will increase and may become the limiting factor for such missions. Here, the dosimetric quantities recommended by the National Council on Radiation Protection and Measurements (NCRP) for the evaluation of health risk due to radiation exposure, effective dose and gray-equivalent to eyes, skin, and blood forming organs (BFO), are calculated for several near Earth environments. These radiation protection quantities are evaluated behind two different shielding materials, aluminum and polyethylene. Since exposure limits for missions beyond low Earth orbit (LEO) have not yet been defined, results are compared to limits recommended by the NCRP for LEO operations.
\end{abstract}

\section{Nomenclature}

$D \quad=$ dose

$E \quad=$ effective dose

$H \quad=$ dose equivalent calculated with a quality factor, $Q$

$H_{T} \quad=$ equivalent dose calculated with radiation weighting factors, $w_{r}$

$\mathrm{L} \quad=$ linear energy transfer

$Q \quad=$ quality factor for stochastic biological effects

$w_{r} \quad=$ radiation weighting factor

$\mathrm{RBE}=$ relative biological effectiveness number

\section{Introduction}

$\mathrm{W}$ HEN evaluating health risk to astronauts due to radiation exposure, there are five requirements. The first is a description of the radiation environment external to the spacecraft or habitat. This description must include the types of particles present (charged ions, neutrons, and/or electrons) and their energy spectra. The second is an accurate model of the shielding provided by the vehicle or habitat. For surface habitats, the protection provided by the planetary surface would be included in the shielding model. The third requirement is a method of calculating the way that the shielding material alters the radiation environment, a radiation transport code. The fourth is a set of dosimetric codes to convert the interior radiation environment into radiation protection quantities, which can be used

\footnotetext{
${ }^{*}$ Physicist, Analytical and Computational Methods Branch, M.S. 188B.

${ }^{\dagger}$ Senior Research Scientist, Analytical and Computational Methods Branch, M.S. 188B.

* Senior Scientist, Wyle/HAC/37A, 1290 Hercules Drive.

$\S$ Aerospace Engineer, Structural and Thermal Analysis Branch, M.S. 431.

${ }^{* *}$ Research Professor, Mathematics Department.
} 
to evaluate astronaut risk. This requirement is the primary focus of this paper. Finally, the fifth requirement is a set of guidelines defining how much radiation is too much, i.e. exposure limits. The creation of tools to rapidly provide spacecraft designers and mission planners with each of these requirements will allow the optimization of radiation shielding to be part of the design process, minimizing shielding mass and cost.

Codes to calculate gray-equivalent and effective dose, the two new radiation protections quantities defined by the NCRP for evaluation of radiation exposure, have recently been completed at NASA Langley Research Center. These codes can be used as stand alone packages, requiring only radiation particle spectra as their input, or they can be used with the HZETRN ${ }^{1}$ space radiation transport code as part of a total radiation analysis tool package. Here environment codes are used to predict the radiation environment for a particular mission. These environments are dependent upon both time in the solar cycle and position of the vehicle or habitat in space. Once the environment has been produced, it is input into the HZETRN code, which produces a data array of particle spectra for varying amounts of shielding material. The particle spectra are then converted into either gray-equivalent or effective dose using the new dosimetry codes. Then a vehicle geometry code can be used to ray trace the spacecraft for any point inside it. This code will calculate how much shielding the vehicle provides in every direction. The dose versus depth data can then be interpolated to calculate the exposure an astronaut inside the vehicle would receive. While these calculations cannot yet be performed in real time, they can be performed in a matter of hours and thus could be very helpful for spacecraft designers and mission planners.

\section{Radiation Protection Quantities}

Once the radiation environment inside a spacecraft or habitat has been calculated, the simplest measure of the damaging effects of this environment is dose, $D$. Dose is the energy absorbed per unit mass of material or tissue and is measured in grays $(1 \mathrm{~Gy}=100 \mathrm{rad}=1 \mathrm{~J}$ per $\mathrm{kg})$. While dose is often used to evaluate risk to electronic equipment and sometimes used to evaluate risk to humans due to the availability of detector data, it does not take into account the varying efficiency of different types of radiation in producing biological effects. For this reason, the quantity dose equivalent, $H$, was defined. Dose equivalent is the product of a quality factor, $Q$, for stochastic effects and the dose at a given point in human tissue, as show in equation (1).

$$
H=\int Q(L) D(L) d L
$$

Note that $Q$ is a function of the linear energy transfer, LET or $L$ in the equation. The quality factor recommended in the International Commission for Radiological Protection (ICRP) report no. $60^{2}$ and adopted by the $\mathrm{NCRP}^{2}$ is shown in equation (2).

$$
\begin{array}{ll}
Q(L)=1, & L<10 \\
Q(L)=0.32 L-2.2, & 10<L<100 \\
Q(L)=300 L^{-1 / 2}, & L>100
\end{array}
$$

Here the LET is in $\mathrm{keV} / \mu \mathrm{m}$ and dose equivalent is expressed in sieverts $(1 \mathrm{~Sv}=100 \mathrm{rems})$. It should be noted that the ICRP ${ }^{2}$ and the $\mathrm{NCRP}^{3}$ also defined a similar protection quantity, equivalent dose denoted $H_{T}$, which makes use of radiation weighting factors, $w_{r}$, instead of the quality factor $Q$. In this work, however, dose equivalent as defined in equations (1) and (2) is used.

Before the publication of NCRP report no. $132^{4}$, the exposure limits for astronaut operations in low earth orbit, LEO, were defined solely in terms of dose equivalent or equivalent dose. The quality factor, Q, used to calculate dose equivalent and the weighting factors, $w_{r}$, were developed from experimental cell death and mutation data to model stochastic effects and are not ideal for modeling deterministic effects. For this reason, a new protection quantity, gray-equivalent, was introduced. To calculate gray-equivalent for a specific organ or tissue site, the radiation environment external to the body is modified by appropriate relative biological effect, RBE, numbers. Then, the dose at that organ or tissue site is evaluated. This is accomplished by calculating the transport of the particles making up the modified environment through the varying amounts of tissue surrounding the dose point. In this work, the HZETRN transport code is used for this purpose. The RBE numbers defined by the NRCP are given in table 1. 
Table 1. RBE numbers for calculating gray-equivalent

\begin{tabular}{|c|c|}
\hline Particle Type & RBE \\
\hline 1 to 5 MeV neutrons & 6.0 \\
\hline 5 to 50 MeV neutrons & 3.5 \\
\hline Heavy ions (e.g. helium, carbon, neon, argon) & 2.5 \\
\hline Protons $>2 \mathrm{MeV}$ & 1.5 \\
\hline
\end{tabular}

Note that RBE numbers have not been defined for neutrons of less than $5 \mathrm{MeV}$ or more than $50 \mathrm{MeV}$. NASA Langley Research Center codes use 5 for low energy neutrons and 3.5 for high energy neutrons ${ }^{5}$. RBE numbers have also not been defined for low energy protons, but this is unnecessary because these protons will not penetrate far into tissue.

NCRP report no. 132 also designated the quality factor effective dose, $E$, to replace dose equivalent as the limiting factor for evaluating risk due to stochastic effects. Effective dose is a weighted average of the dose equivalent to a number of organs and tissues sites and is given by equation (3).

$$
E=\Sigma w_{T} H_{T}
$$

where the weight factors, $w_{T}$, are given in table 2.

Table 2. Weight factors, $w_{T}$, for calculating effective dose.

\begin{tabular}{|c|c|c|c|}
\hline $\mathbf{0 . 0 1}$ & $\mathbf{0 . 0 5}$ & $\mathbf{0 . 1 2}$ & $\mathbf{0 . 2 0}$ \\
\hline Bone surface & Bladder & Bone marrow & Gonads \\
\hline skin & Breast & Colon & \\
\hline & Liver & Lung & \\
\hline & Esophagus & Stomach & \\
\hline & Thyroid & & \\
\hline & Remainder & & \\
\hline
\end{tabular}

\section{Exposure Limits}

The most important thing to note is that as yet, no exposure limits have been set for missions beyond LEO. Therefore, free space exposure rates will be compared to the limits for LEO operations. For LEO operations, in addition to a federally mandated obligation to follow the principle of keeping exposure as low as reasonably achievable (ALARA), NASA has adopted and OSHA has approved the radiation exposure recommendations introduced in NCRP report no. $132^{4}$. The NCRP has set the monthly, annual, and career exposure limits shown in tables 3 and 4.

Table 3. LEO exposure limits for blood forming organs, eyes, and skin for all ages.

\begin{tabular}{|l|c|c|c|}
\hline & $\begin{array}{c}\text { BFO } \\
(\mathrm{Gy}-\mathrm{Eq})\end{array}$ & $\begin{array}{c}\text { Eye } \\
(\mathrm{Gy}-\mathrm{Eq})\end{array}$ & $\begin{array}{c}\text { Skin } \\
(\mathrm{Gy}-\mathrm{Eq})\end{array}$ \\
\hline Career & See Table 4 & 4.00 & 6.00 \\
\hline Annual & 0.50 & 2.00 & 3.00 \\
\hline 30 Day & 0.25 & 1.00 & 1.50 \\
\hline
\end{tabular}

Table 4. LEO career whole body effective dose limits (Sv).

\begin{tabular}{|c|c|c|c|c|}
\hline Age & 25 & 35 & 45 & 55 \\
\hline Male & 0.7 & 1.0 & 1.5 & 3.0 \\
\hline Female & 0.4 & 0.6 & 0.9 & 1.7 \\
\hline
\end{tabular}

These career limits are based on a requirement that there be no more than $3 \%$ excess career fatal cancer risk. 


\section{Protection Quantity Calculations}

The exposure rates for a number of environments have been calculated and are shown in figures 1-7. Here the Badhwar-O'Neill model of the 1977 solar minimum environment ${ }^{6}$ was used as a maximum or "worst case" GCR environment. The GCR environment is made up of charged ions entering the solar system from outside. For this reason, the GCR environment is most intense when the solar wind is at a minimum.

Figure 1 shows the effective dose rates for free space. While these daily numbers appear to be exceptionally small, it is important to note that if a manned mission to Mars were to last 2 years, astronauts would receive in excess of $1 \mathrm{~Sv}$ even if the vehicle provided $10 \mathrm{~g} / \mathrm{cm}^{2}$ of aluminum shielding. Thus younger astronauts would be unable to perform this mission, if the LEO limits are used. Figure 1 also demonstrates the advantages of using shielding material with high hydrogen content. Effective dose rate behind polyethylene shielding are much lower.

Figure 2 compares the effective dose rates behind polyethylene shielding for two different environments, solar maximum and solar minimum. The BadhwarO'Neill model is also used for the 1990 solar maximum, a "best case" scenario. This figure demonstrates the advantage of performing missions during solar maximum.

Figure 3 shows daily gray-equivalent values for the 1977 solar minimum. Clearly, astronauts are not in danger of reaching the 30-day or annual limits given in table 3 due to GCR exposure. Therefore, only the career effective dose limits in table 4 are of concern for GCR radiation.

Figures 4 and 5 shows the effective dose and grayequivalent that a male astronaut would receive if exposed to a solar particle event equivalent to four times the intensity of the September 28, 1989 event. This spectrum closely approximates the 99-percentile event. ${ }^{7}$ Solar particle events occur when a large number of particles, primarily protons, move through the solar system. These events happen during periods of increased solar activity and appear to correspond to large coronal mass ejections. ${ }^{8}$ Large solar particle events are extremely rare and last only a matter of hours. In the last fifty years, we have had only one or two per eleven-year solar cycle. However, if astronauts are not adequately shielded, exposure to a large SPE could result in extreme radiation sickness or lethality.

Because solar particle events are of short duration, usually less than a day, shielding could be provided by a storm shelter that the astronauts stay in for the duration of the event or by personal shielding devices donned by the astronauts.

Astronauts performing surface operations receive shielding in $2 \pi$ directions from the planetary surface. There are, however, some backscattered particles produced in the surface materials when GCR ions interact with them, but these are mostly low energy neutrons and a small number of protons, and they contribute little to dose. For this reason, dose rates on the surface of the moon, which are not shown here, are slightly more than half those of free space. The Martian atmosphere provides additional shielding, so dose rates are smaller. These exposure rates are shown in figures 6 and 7.

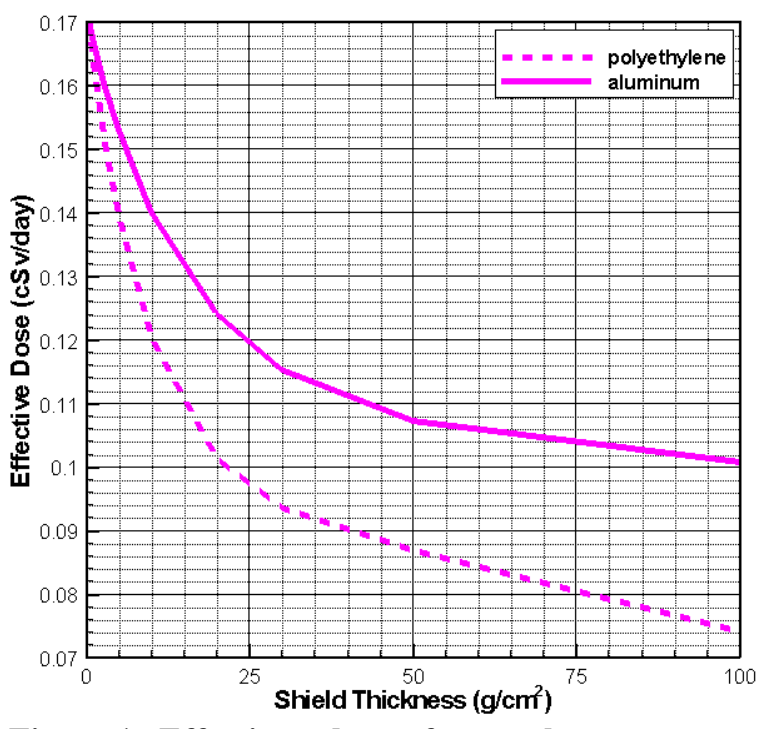

Figure 1. Effective dose for male astronauts behind polyethylene or aluminum spherical shielding exposed to the free space 1977 solar minimum GCR environment.

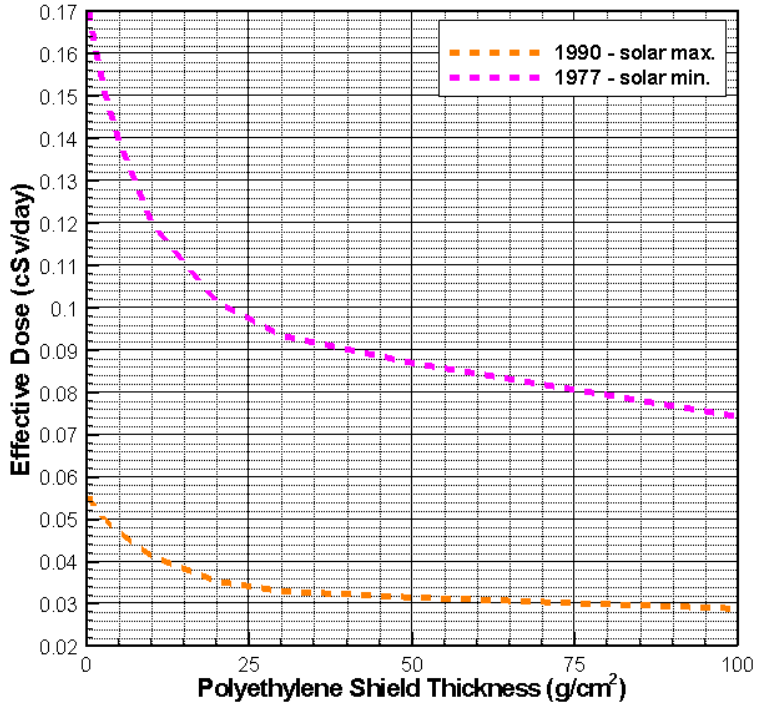

Figure 2. Effective dose for male astronauts behind polyethylene spherical shielding exposed to the free space 1977 solar minimum or 1990 solar maximum GCR environment. 


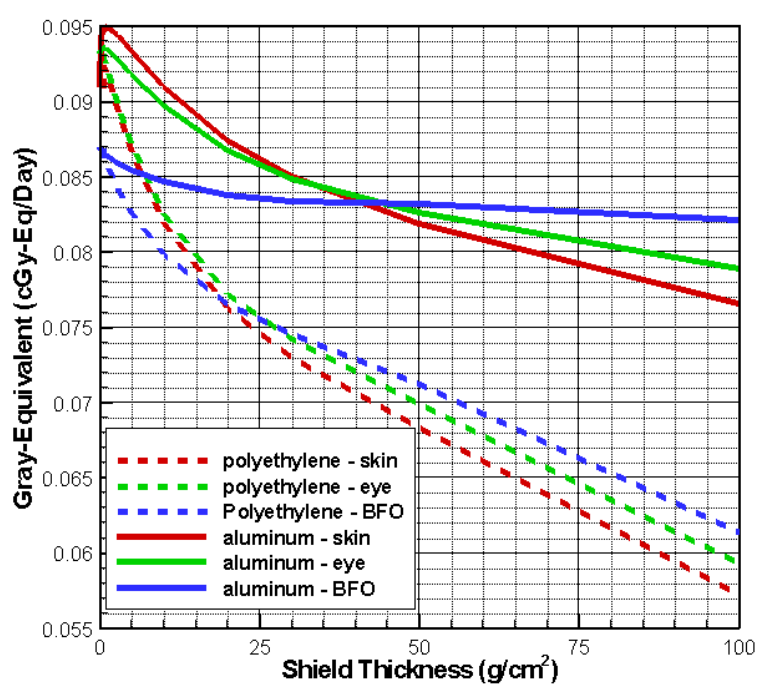

Figure 3. Gray equivalent to skin, eyes, and blood forming organs behind polyethylene or aluminum spherical shielding exposed to the free space 1977 solar minimum GCR environment.

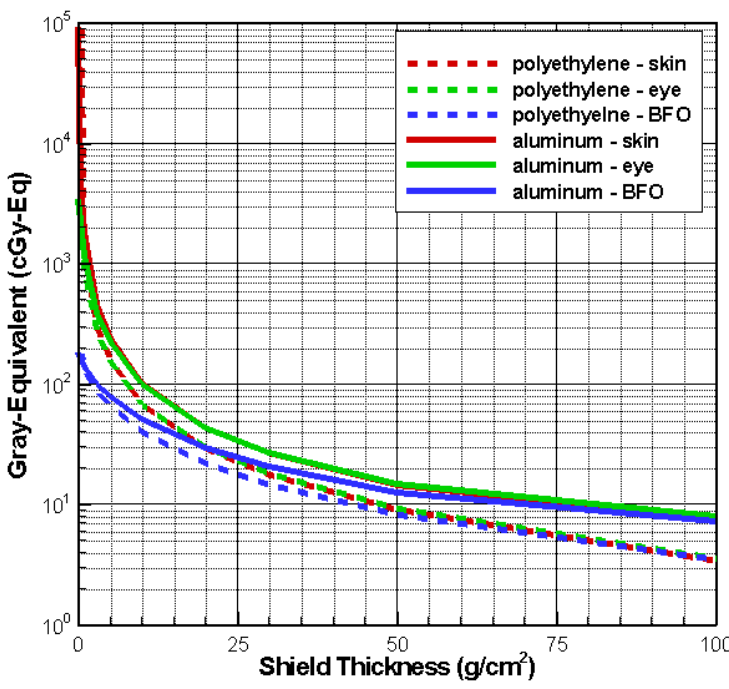

Figure 5. Gray equivalent to skin, eyes, and blood forming organs behind polyethylene or aluminum spherical shielding exposed to 4 times the September 1989 solar particle event.

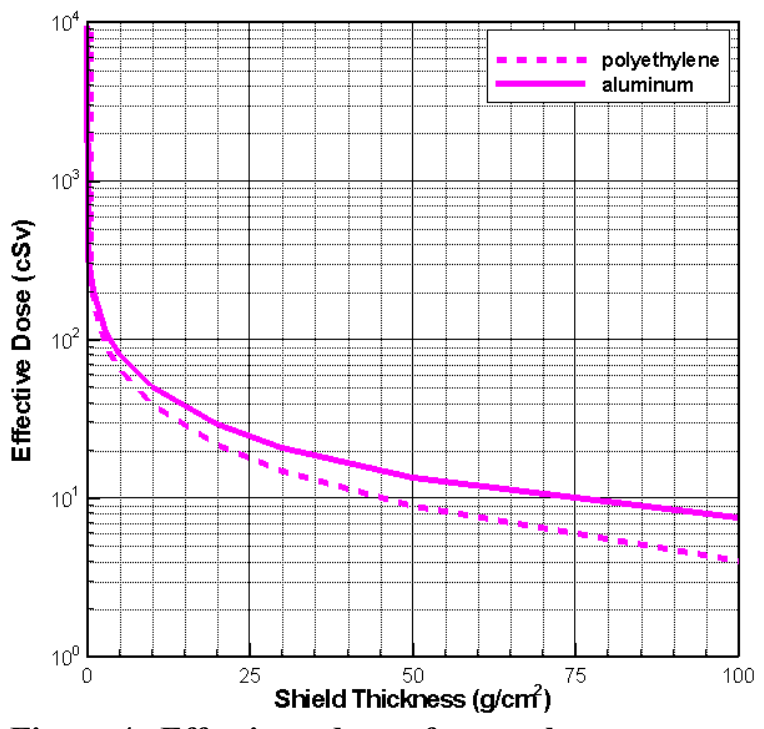

Figure 4. Effective dose for male astronauts behind polyethylene or aluminum spherical shielding exposed to 4 times the September 1989 solar particle event.

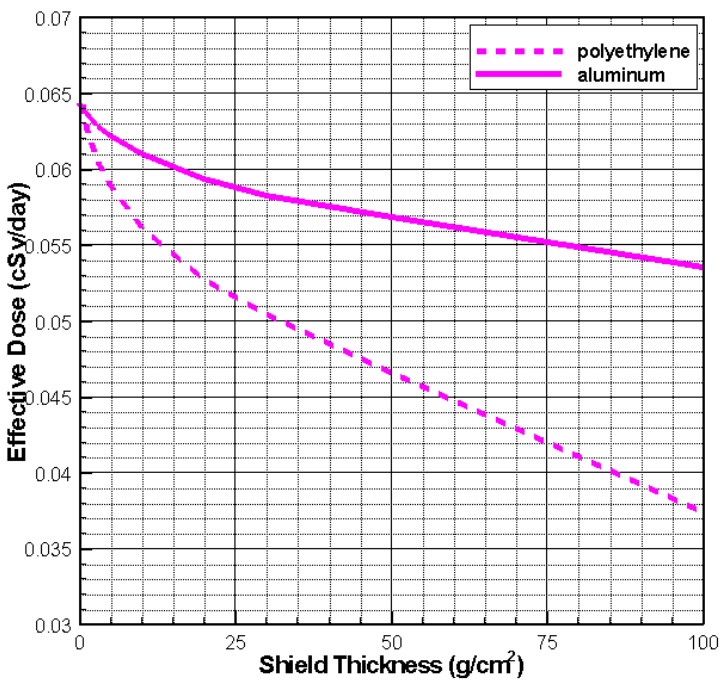

Figure 6. Effective dose for male astronauts behind polyethylene or aluminum spherical shielding on the surface of Mars during the 1977 solar minimum GCR environment.

\section{Concluding Remarks}

Codes to rapidly calculate gray-equivalent and effective dose have been developed. These codes can be used with environmental models and a radiation transport code to evaluate shielding requirements for a variety of space exploration missions. Preliminary calculations show that if exposure limits for long-term missions beyond LEO are similar to those for LEO, radiation shielding could become the limiting factor. The calculations show that astronauts must be provided with a "storm shelter" in case a large solar particle event occurs. Further, the importance of choosing structural and shielding materials with high hydrogen content is emphasized. 


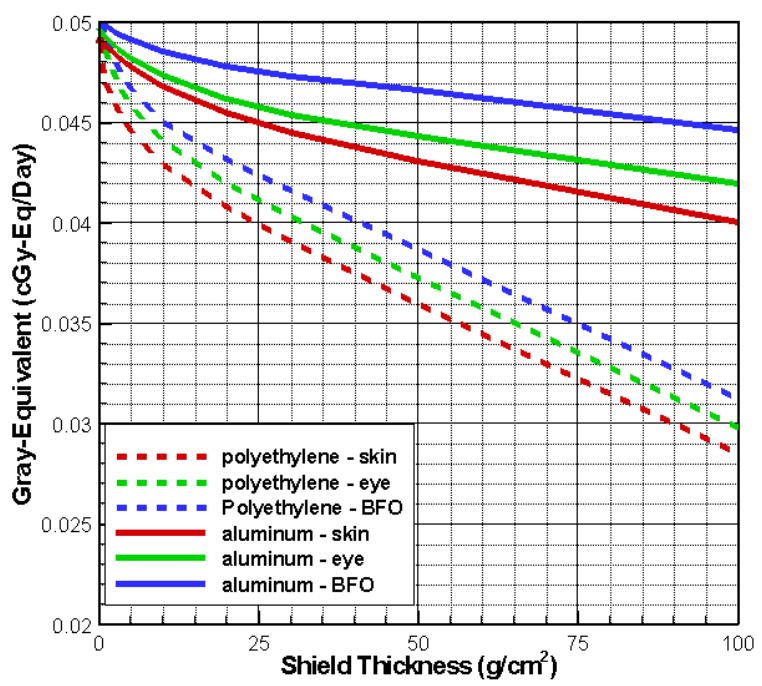

Figure 7. Gray equivalent to skin, eyes, and blood forming organs behind polyethylene or aluminum spherical shielding on the surface of Mars during the 1977 solar minimum GCR environment.

\section{References}

${ }^{1}$ Wilson, J. W., Kim, M. Y., De Angelis, G., Cucinotta, F. A., Yoshizawa, N., and Badavi, F. F., "Implementation of Gy-Eq for Deterministic Effects Limitation in Shield Design," J. Radiat. Res., 43, suppl. pp. S103-S106, 2002.

" "1990 Recommendations of the International Commission for Radiological Protection," ICRP Report No. 60, Annuals of the ICRP 21, No. 1-3, Elsevier Science, New York, 1991.

3"Limitation of Exposure to Ionizing Radiation," NCRP Report No. 116, National Council on Radiation Protection and Measurements, Bethesda, MD, 2001.

4"Radiation Protection Guidance for Activities in Low-Earth Orbit," NCRP Report No. 132, National Council on Radiation Protection and Measurements, Bethesda, MD, 2001.

${ }^{5}$ Wilson, J. W., Badavi, F. F., Cucinotta, F. A., Shinn, J. L., Badhwar, G. D., Silberberg, R., Tsao, C. H., Townsend, L. W., and Tripathi, R. K., "HZETRN: Description of a Free-Space Ion and Nucleon Transport and Shielding Computer Program," NASA TP-3495, 1995.

${ }^{6}$ Badhwar, G. D., and O’Neill, P. M., Nucl. Tracks and Rad. Meas., 20, pp. 403-410, 1992.

${ }^{7}$ Xapsos, M. A., Barth, J. L., Stassinopoulos, E. G., Burke, E. A., and Gee, G. B., "Space Environment Effects: Model for Emission of Solar Protons (ESP) - Cumulative and Worst-Case Event Fluences," NASA TP-1999-209763, 1999.

${ }^{8}$ Reames, D. V., Space Sci. Rev., 90, pp. 417-491, 1999. 